\title{
Fine-scale differentiation between Bacillus anthracis and Bacillus cereus group signatures in metagenome shotgun data
}

\author{
Robert A Petit III ${ }^{1}$ ， James M. Hogan ${ }^{2}$ ， Matthew N. Ezewudo ${ }^{1}$ ， Sandeep J. Joseph ${ }^{1}$ ， Timothy D. Read ${ }^{\text {Corresp. } 1}$ \\ 1 Department of Medicine, Division of Infectious Diseases, Emory University School of Medicine, Atlanta, Georgia, United States \\ 2 Queensland University of Technology, Brisbane, Australia \\ Corresponding Author: Timothy D. Read \\ Email address: tread@emory.edu
}

\section{Background}

It is possible to detect bacterial species in shotgun metagenome datasets through the presence of only a few sequence reads. However, false positive results can arise, as was the case in the initial findings of a recent New York City subway metagenome project. False positives are especially likely when two closely related are present in the same sample. Bacillus anthracis, the etiologic agent of anthrax, is a highconsequence pathogen that shares $>99 \%$ average nucleotide identity with Bacillus cereus group (BCerG) genomes. Our goal was to create an analysis tool that used k-mers to detect $B$. anthracis, incorporating information about the coverage of BCerG in the metagenome sample.

\section{Methods}

Using public complete genome sequence datasets, we identified a set of 31-mer signatures that differentiated $B$. anthracis from other members of the $B$. cereus group (BCerG), and another set which differentiated BCerG genomes (including $B$. anthracis) from other Bacillus strains. We also created a set of 31-mers for detecting the lethal factor gene, the key genetic diagnostic of the presence of anthraxcausing bacteria. We created synthetic sequence datasets based on existing genomes to test the accuracy of a k-mer based detection model.

\section{Results}

We found 239,503 B. anthracis-specific 31-mers (the Ba31 set), 10,183 BCerG 31-mers (the BCerG31 set), and 2,617 lethal factor k-mers (the lef31 set). We showed that false positive $B$. anthracis k-mers which arise from random sequencing errors - are observable at high genome coverages of $B$. cereus. We also showed that there is a "gray zone" below $0.184 x$ coverage of the $B$. anthracis genome sequence, in which we cannot expect with high probability to identify lethal factor k-mers. We created a linear regression model to differentiate the presence of $B$. anthracis-like chromosomes from sequencing errors given the BCerG background coverage. We showed that while shotgun datasets from the New York City subway metagenome project had no matches to lef31 k-mers and hence were negative for $B$. anthracis, some samples showed evidence of strains very closely related to the pathogen.

\section{Discussion}

This work shows how extensive libraries of complete genomes can be used to create organism-specific signatures to help interpret metagenomes. We contrast "specialist" approaches to metagenome analysis such as this work to "generalist" software that seeks to classify all organisms present in the sample and note the more general utility of a k-mer filter approach when taxonomic boundaries lack clarity or high 
levels of precision are required. 
1 Fine-scale differentiation between $B$. anthracis

2 and $B$. cereus group signatures in metagenome

3 shotgun data

4

5 Robert A. Petit III' , James M. Hogan², Matthew N. Ezewudo ${ }^{1}$ Sandeep J. Joseph ${ }^{1}$, 6 Timothy D. Read ${ }^{1 *}$

7

$8{ }^{1}$ Division of Infectious Diseases, Department of Medicine, Emory University School of Medicine,

9 Atlanta, Georgia, USA.

10

$11{ }^{2}$ Queensland University of Technology, Brisbane, Australia.

12

13

14

15

16 


\section{Abstract}

18 Background

19 It is possible to detect bacterial species in shotgun metagenome datasets through the presence of only a few sequence reads. However, false positive results can arise, as was the case in the initial findings of a recent New York City subway metagenome project. False positives are especially likely when two closely related are present in the same sample. Bacillus anthracis, the etiologic agent of anthrax, is a high-consequence pathogen that shares $>99 \%$ average nucleotide identity with Bacillus cereus group (BCerG) genomes. Our goal was to create an analysis tool that used k-mers to detect $B$. anthracis, incorporating information about the coverage of BCerG in the metagenome sample.

Methods

Using public complete genome sequence datasets, we identified a set of 31-mer signatures that differentiated $B$. anthracis from other members of the $B$. cereus group (BCerG), and another set which differentiated BCerG genomes (including $B$. anthracis) from other Bacillus strains. We also created a set of 31-mers for detecting the lethal factor gene, the key genetic diagnostic of the presence of anthrax-causing bacteria. We created synthetic sequence datasets based on existing genomes to test the accuracy of a k-mer based detection model.

\section{Results}

We found 239,503 B. anthracis-specific 31-mers (the Ba31 set), 10,183 BCerG 31-mers (the $B C$ erG31 set), and 2,617 lethal factor k-mers (the lef31 set). We showed that false positive $B$. anthracis k-mers - which arise from random sequencing errors - are observable at high genome coverages of $B$. cereus. We also showed that there is a "gray zone" below $0.184 x$ coverage of the $B$. anthracis genome sequence, in which we cannot expect with high probability to identify lethal factor k-mers. We created a linear regression model to differentiate the presence of $B$. anthracis-like chromosomes from sequencing errors given the BCerG background coverage. We showed that while shotgun datasets from the New York City subway metagenome project had no matches to lef31 k-mers and hence were negative for $B$. anthracis, some samples showed evidence of strains very closely related to the pathogen.

\section{Discussion}

This work shows how extensive libraries of complete genomes can be used to create organismspecific signatures to help interpret metagenomes. We contrast "specialist" approaches to metagenome analysis such as this work to "generalist" software that seeks to classify all organisms present in the sample and note the more general utility of a k-mer filter approach when taxonomic boundaries lack clarity or high levels of precision are required. 


\section{Introduction}

53 There is great interest in the use of shotgun metagenome data to detect pathogens in clinical 54 and environmental samples. A large number of bioinformatic tools have been developed 55 (Mclntyre et al., 2017) that use different algorithmic approaches to rapidly parse and analyze 56 sequence data files. Over the last 8-10 years, these data have been generated primarily by 57 Illumina sequencing technology. Typically, sequences from metagenomic data files are matched 58 against public reference databases, such as NCBI RefSeq. Consistency of matches across the 59 tree of life is dependent therefore on the database entries being correctly labelled, having 60 similar levels of representation across species, and having species defined in a consistent 61 manner. However, we are beginning to understand how the skewed representation of taxa contained in the database sometimes affects sampling accuracy (Nasko et al., 2018). The classification of many bacterial species harks back to distinctions based on morphological, biochemical and virulence characteristics, made prior to the advent of DNA sequencing. Sometimes, unusually close species boundaries can confound metagenomic classifiers and result in false positive matches. In 2015, Afshinnekoo et al (Afshinnekoo et al., 2015a) published initial findings from an extensive study of the New York Subway metagenome, which claimed that they had detected bacteria responsible for anthrax (Bacillus anthracis) and plague (Yersinia pestis). While these misidentifications were swiftly corrected (Mason, 2015; Afshinnekoo et al., $2015 b$ ), indistinct or fuzzy boundaries between species may yield many errors of this nature.

$B$. anthracis, the pathogen that is the focus of this work, is a Gram-positive bacterium that forms tough endospores allowing it to survive dormant in the environment for years. The $5.2(\mathrm{Mbp})$ main chromosome shares an average nucleotide identity (ANI, (Konstantinidis \& Tiedje, 2005) in excess of $99 \%$ with other members of the collection of species known as the 'Bacillus cereus group' (BCerG)(Helgason et al., 2000). The most common species in this group are $B$. cereus, $B$. thuringiensis and B. mycoides (Helgason et al., 2000; Zwick et al., 2012). The recommended level of difference between bacterial species is an ANI of 95\% (Konstantinidis \& Tiedje, 2005). While BCerG strains are mostly opportunistic pathogens of invertebrates and are commonly found in soil, B. anthracis kills mammals (Carlson et al., 2018). Spores are generally found at high titers in soils where animals have recently died from anthrax. Phylogeographic analysis has shown that $B$. anthracis is probably native to Africa, with only recent transfer of a limited number of lineages to other continents (Keim \& Wagner, 2009). For these reasons, it would be an unusual outcome to find spores in the New York subway(Ackelsberg et al., 2015).

What sets $B$. anthracis apart from other BCerG strains is the presence of two plasmids: pXO1 (181 kb), which carries the lethal toxin genes, and pXO2 (94 kb), which includes genes for a protective capsule. Without either of these plasmids, $B$. anthracis is considered attenuated in virulence and unable to cause classic anthrax (Dixon et al., 1999). Plasmids from other BCerG genomes may be very similar to pXO1 and pXO2 but lack the important virulence genes (Rasko et al., 2007). Rarely, BCerG strains carry pXO1 and appear to cause anthrax-like disease (Hoffmaster et al., 2004; Hoffmann et al., 2017); pXO2-like plasmids are also quite common in BCerG and other Bacillus species(Pannucci et al., 2002; Cachat et al., 2008). 
95

96

97

98

99

100

101

102

103

104

105

106

107

108

109

110

111

112

113

114

115

116

117

118

119

120

121

122

123

124

125

126

127

128

129

130

131

132

133

134

135

136

Shortly after the release of the NYC subway metagenome paper, we produced a blog post (Petit et al., 2015) that critically re-analyzed these data in the light of what was known about $B$. anthracis genomics. This work, and other critiques, led to reassessment of the data and revisions to the original manuscript. In this paper, we incorporate some of the results introduced informally on our blog and extend them to create a k-mer based approach - using recent public $B$. anthracis and BCerG data - to analyze in greater detail how to search for traces of $B$. anthracis in shotgun metagenome data. While elements of this method are necessarily specific to $B$. anthracis and the context of the BCerG group, the general strategy has far broader utility and this work is a model for future "specialist" studies based on k-mer filtering.

\section{Methods}

Metagenome data and reference genome sequences

Shotgun metagenomic data from the "NYC" study SRP051511 (Afshinnekoo et al., 2015a) were downloaded from the Sequence Read Archive (SRA) with sra-tools (fastq-dump -I \$SRA_ACCESSION, v2.8.2, https://github.com/ncbi/sra-tools). Reference genomes for different taxonomic groups were downloaded from the NCBI Nucleotide database in April 2018 with the following queries:

All BCerG genomes = 'txid86661[Organism:exp] AND "complete genome"[Title] AND refseq[filter] AND 3000000:7000000[Sequence Length]'

All non-BCerG Bacillus genomes = 'txid1386[Organism:exp] NOT txid86661[Organism:exp] "complete genome"[Title] AND 3000000:7000000[Sequence Length] AND refseq[filter]'

Bacillus anthracis genomes were included in the BCerG genome query. The lethal factor gene was extracted from completed pXO1 plasmids downloaded with the following query:

pXO1 plasmid = 'pXO1[Title] AND 140000:200000[Sequence Length] ‘

The results of these queries, as of April 2018, are available on our git repository.

Mapping metagenome data to $B$. anthracis plasmids and chromosomes

$B$. anthracis positive samples and control samples were mapped against reference pXO1 (CP009540) and pXO2 (NC_007323) plasmids and reference B. anthracis (CP009541) and B. cereus (NC_003909) completed genomes with BWA (bwa mem -t \$NUM_CPU \$REFERENCE \$FASTQ_R1 \$FASTQ_R2 > \$SAM_FILE, v0.7.5a-r405, (Li \& Durbin, 2009)). The aligned reads in SAM format were converted to sorted BAM and indexed with SAMtools (samtools view -@ 10 bS \$SAM_FILE | samtools sort -@ 10 - \$SAMPLE, v1.1, (Li et al., 2009)). The per base coverage was extracted with genomeCoverageBed from BEDTools (genomeCoverageBed -d ibam \$BAM_FILE | gzip --best - > \$COVERAGE, v2.16.2, (Quinlan \& Hall, 2010)). Coverage across the plasmids and chromosomes was plotted for multiple sliding windows with a custom Rscript. Mapped reads were extracted and saved in FASTQ with bam2fastq (bam2fastq -o 
138 https://gsl.hudsonalpha.org/information/software/bam2fastq) and FASTA format with fastq_to_fasta from FASTX Toolkit (cat \$FASTQ_FILE | fastq_to_fasta -Q33 -n | gzip -best - > \$FASTA_OUTPUT, v0.0.13.2, (Gordon \& Hannon, 2010)). Scripts, runtime parameters, and output are available at this site (Petit et al., 2015): https://github.com/Read-LabConfederation/nyc-subway-anthrax-study.

143

Custom 31-mer assay for B. anthracis and Bacillus cereus Group database as not being part of the BCerG (see the Results section). To create a rational method to assign taxonomy to genomes for this study we used mash (mash sketch -k 31 - s 100000 - $p$ \$NUM_CPU -o \$OUTPUT_PREFIX *.fasta, V2.0, (Ondov et al., 2016)) to reclassify mislabeled Bacillus genomes as $B$. anthracis, non-anthracis BCerG, or non-BCerG. We identified Bacillus anthracis strain 2002013094 (NZ_CP009902) as the most distant (Mash distance 0.000687) B. anthracis member from B. anthracis str. Ames (NC_003997). We also identified Bacillus cytotoxicus NVH 391-98 (NC_009674) as the most distant (Mash distance 0.135333) BCerG member from B. anthracis str. Ames (NC_003997). We then determined the Mash distance of all Bacillus genomes from $B$. anthracis str. Ames (mash dist - $p$ \$NUM_CPU \$MASH_SKETCH $\$$ \$FASTA_FILE). We used the Mash distance to reclassify each Bacillus genome as $B$. anthracis (Mash distance $<=0.000687$ ), non-anthracis BCerG (Mash distance $<=0.135333$ ), or nonBCerG (Mash distance $>0.135333$ ). A phylogeny of all completed Bacillus genomes was created with mashtree (mashtree --numcpus $20 *$.fasta > bacillus-mashtree.dnd, v0.32, https://github.com/lskatz/mashtree).

160

Sequence 31-mers were extracted and counted with Jellyfish (jellyfish count - $C$-m 31 -s $1 \mathrm{M}$-O \$JELLYFISH_DB \$FASTA_FILE, V2.2.3, (Marçais \& Kingsford, 2011)) and partitioned into two distinct sets characteristic of BCerG (BCerG31) and B. anthracis (Ba31) (Figure 1). The BCerG31 and Ba31 sets were initially comprised of 31-mers conserved within every member of BCerG (including $B$. anthracis) and those restricted to only $B$. anthracis, respectively. Any Ba31mers found in non-anthracis BCerG members or non-BCerG genomes were filtered out. Likewise, any BCerG31-mers found in non-BCerG Bacillus genomes were filtered out. 31-mers found in rRNA were filtered out with a Jellyfish database created from the SILVA rRNA database (Quast et al., 2013). We further filtered the Ba31 and BCerG31 sets using the nonredundant nucleotide sequence database (NT v5, downloaded April 2017). We used BLASTN (blastn -max_hsps 1 -max_target_seqs 1 -dust no -word_size 7 -outfmt 15 -query \$FASTA_FILE -db \$BLAST_DB - evalue 10000 -num_threads \$NUM_CPU, v2.8.0, (Camacho et al., 2009)) to align Ba31 against non-anthracis BCerG sequences and BCerG31 against non-BCerG sequences. 31-mers with an exact match were filtered out.

175

176 Finding the limits for lethal factor-based detection of $B$. anthracis

177 We used $B$. anthracis whole genome shotgun sequencing projects to determine the limit of 178 detection of lethal factor k-mers (lef31). We defined lef31 as the unique set of 31-mers identified 
179 in lef genes downloaded from the NCBI Nucleotide database (previously described) (Figure 1).

180

181

182

183

184

185

186

187

188

189

190

191

192

193

194

195

196

197

198

199

200

201

202

203

204

205

206

207

208

209

210

211

212

213

214

215

216

217

218

219

220

221

$B$. anthracis projects were identified from the SRA with the following query:

$B$. anthracis projects = "genomic[Source] AND random[Selection] AND

txid86661[Organism:exp] AND paired[Layout]) AND wgs[Strategy] AND "Illumina HiSeq"'

In this work we have assumed a 95\% 'confidence limit' for detection of the lethal factor k-mers, so that detection is held to fail if fewer than $95 \%$ of a set of random subsamples are found to contain at least one lethal factor k-mer. The threshold is then obtained through computational experiment. For each project, we started at $0.2 \times B$. anthracis genome coverage and extracted 100 random subsamples of sequences, using Jellyfish as before to determine if at least one lethal factor k-mer was present. We then continued this process, reducing the coverage until fewer than $95 \%$ of the subsamples contained at least one lethal factor k-mer. The previous coverage was then recorded as the limit of detection of the lethal toxin for a given sample.

\section{Assessing Quality of B. anthracis and Bacillus cereus Group specific 31-mers}

We used ART (art_illumina - 1100 - $f$ \$COVERAGE -na - $S S$ HS20 -rs \$RANDOM_SEED - $i$ \$FASTA_FILE - o \$OUTPUT_PREFIX, vMountRainier-2016-06-05, (Huang et al., 2012)) to simulate 100 bp reads with the built-in Illumina HiSeq 2000 error model for each non-anthracis Bacillus genome. The Illumina HiSeq 2000 error model was selected to match the predominant sequencing technology of the NYC dataset. We simulated coverages ranging from $0.01 \mathrm{x}$ to $15 \mathrm{x}$ to determine if false positive Ba31 matches were uniform across non-anthracis BCerG members. We counted 31-mers for each simulated read set with Jellyfish as previously described. We then used the k-mer counts to determine the false positive Ba31 counts in nonanthracis genomes. We found the false positive Ba31 counts to be higher in non- $B$. anthracis genomes that were most closely related to $B$. anthracis (please see results section). A subset of non- $B$. anthracis BCerG genomes with a Mash distance less than 0.01 from $B$. anthracis, previously described, were selected as our model set. We further simulated coverages from $15 \mathrm{x}$ to $100 x$ to match levels of coverage observed in the NYC dataset. We then applied linear regression, implemented in the $R$ base stats package ( $R$ v3.4.3), on this subset to develop a predictive model with the observed Ba31 count as our dependent variable and the observed BCerG k-mer coverage as our independent variable.

\section{Prediction of low coverage B. anthracis chromosome in simulated metagenomic} sequencing datasets

We used ART to simulate metagenomic mixtures of $B$. anthracis str. Ames (NC_003997) and $B$. cereus strain JEM-2 (NZ_CP018935). B. cereus strain JEM-2 was selected because it was the closest non-anthracis BCerG member to B. anthracis str. Ames (Mash distance 0.00873073). We used coverages between $0-100 x$ for $B$. cereus and coverages between $0-0.2 x$ for $B$. anthracis. A python script (subsample-ba-lod.py NC_003997.fasta NZ_CP018935.fasta coverages-ba.txt coverages-bcg.txt temp_folder/ fasta/ ba-specific-kmers.fasta bcgspecific-kmers.fasta) was created to simulate mixtures for each pairwise combination of $B$. cereus and $B$. anthracis coverages. For each mixture, we determined the Ba31 and BCerG31 
222

223

224

225

226

227

228

229

230

231

232

233

234

235

236

237

238

239

240

241

242

243

244

245

246

247

248

249

250

251

252

253

254

255

256

257

258

259

260

261

counts with Jellyfish as previously described. This process was repeated 20 times per pairwise combination of coverages. The model was applied to determine what level of $B$. anthracis coverage was required to differentiate observed Ba31-mers from sequencing errors.

We determined Ba31, BCerG31 and lef31 counts for each sample in the NYC study. The model was applied to these counts to determine if observed $B$. anthracis k-mers exceeded the level expected due to sequencing errors.

We processed each of the subsampled mixtures and samples from the NYC study with KrakenHLL (krakenhll --report-file \$REPORT_FILE --db \$DATABASE > \$SEQUENCES, v0.4.7, (Breitwieser \& Salzberg, 2018)). We used dustmasker (dustmasker -outfmt fasta, v2.8.0, (Camacho et al., 2009)) to create a DUST-masked version of the standard Kraken database (kraken-build --standard --db \$DATABASE, database built in April 2017) for this analysis. From the final Kraken report, the number of reads and unique k-mers identified for $B$. anthracis were extracted. We compared these results to our method.

Output, figures, runtime parameters and scripts from this study are available in a git repository hosted at: https://doi.org/10.5281/zenodo.1323741.

\section{Results}

NY subway metagenome sequences map to core regions of $B$. anthracis and $B$. cereus chromosome and plasmids but not to lethal factor gene

In the original analysis of the subway metagenome (Afshinnekoo et al., 2015a), two samples (P00134 (SRR1748707, SRR1748708), and P00497 (SRR1749083)) were reported to contain reads that mapped to Bacillus anthracis based on results obtained using the Metaphlan software (Segata et al., 2012). We found that 792,282 reads from P00134 and 270,964 reads from P00497 mapped to the $B$. anthracis strain Sterne chromosome. The reads aligned along the entire length of the chromosome, forming a characteristic peak at the replication origin, a pattern often seen when other bacterial chromosomes have been recovered from metagenome samples (Brown et al., 2016). However, a similar number of reads from P00134 and P00497 (765,466 reads and 265,776 reads, respectively) mapped to the $B$. cereus 10987 chromosome. We also found that P00134 and P00497 reads mapped to the both the pXO1 and pXO2 plasmids in conserved "backbone" regions (Rasko et al., 2007) but that no read mapped to the mobile element containing the lef lethal factor gene. These results showed that the close taxonomic relationship of $B$. anthracis and BCerG made identification of the biothreat agent by mapping reads alone unreliable. In addition, the pXO1 and pXO2 plasmids were not reliable as positive markers for $B$. anthracis at low genome coverages (when the lef gene may not be sampled, see next section) because backbone sequences cross-matched against plasmids found in BCerG strains. 
262

263

264

265

266

267

268

269

270

271

272

273

274

275

276

277

278

279

280

281

282

283

284

285

286

287

288

289

290

291

292

293

294

295

296

297

298

299

300

301

302

303

304

\section{B. anthracis genome coverage below $0.184 x$ is a "gray area" for detection, where} lethal toxin genes may not be sampled

The best test for presence of virulent $B$. anthracis (or virulent $B$. cereus strains containing $\mathrm{pXO1}$ ) is detection of the lethal factor gene (2,346 bp) (Bragg \& Robertson, 1989). However, at low sequence coverage of the pathogen, it is not certain that reads from this gene will be present (given the 3:1 copy number ratio of pXO1 to $B$. anthracis chromosome (Read et al., 2002) the ratio of chromosome to lef is $\sim 620: 1$ ). We identified 2,617 31-mers present in 36 lef gene sequences and called this set "lef31". To estimate the coverage sufficient that we would expect (with probability above some threshold value, here 0.95 ) to observe lethal factor sequences, we randomly subsampled reads from $164 \mathrm{~B}$. anthracis genome projects and tested for the presence of at least one lef31 match (Figure 2). With Ba31 and BCerG31 k-mer coverages below 0.103x and $0.112 x$, respectively, this analysis showed we would have less than a $95 \%$ chance of sampling a single lef31 k-mer even if the lef gene were present. These k-mer coverages are approximately $0.184 x$-fold $B$. anthracis genome coverage, or 9,360100 base pair reads (Supplementary Figure 1).

\section{Conserved and specific 31-mer sets for $B$. anthracis and BCerG chromosomes}

The results of the previous section showed that at low $B$. anthracis genome coverage, detection of the lethal factor is not guaranteed. In metagenomic samples, in which sequencing coverage is expected to be low for rare organisms, the most reliable way to detect $B$. anthracis was to use chromosomal genetic signatures that distinguished the species from close relatives. We identified 239,503 31-mers conserved in $48 \mathrm{~B}$. anthracis reference genomes that were not also detected in the remainder of the Bacillus genus (331 genomes), rRNA sequences, or the BLAST non-redundant nucleotide database. We called this set "Ba31".

We created a second set of 31-mers specific to and conserved in all BCerG genomes (including B. anthracis). Surprisingly, our initial analysis produced zero 31-mers specific to all 139 BCerG strains and not other Bacillus. Inspection of the whole genome phylogeny (Figure 3) showed that 4 genomes (NZ_CP007512, NZ_CP017016, NZ_CP020437, NZ_CP025122) that fell within the BCerG clade based on phylogeny had not been classified as BCerG in the NCBI Taxonomy hierarchy. After reclassifying these strains as BCerG, we identified 10,183 BCerG specific 31mers, which we called "BCerG31".

High background levels of $B$. cereus strains produce false positive $B$. anthracis specific k-mers due to random sequence errors

We simulated synthetic data of Bacillus reference genomes at different genome coverages using ART software with an error model based on Illumina short read data (Huang et al., 2012) (Figure 4). We defined 'k-mer coverage' as the sum of counts for k-mers detected divided by the number of $k$-mers in the k-mer set. Ba31 and BCerG k-mer coverage had a linear relationship with genome coverage (Supplementary Figure 1). The coefficient was less than 1 (0.56 and 0.61 for Ba31 and BCerG31 respectively), because some portions of the chromosomes were not well sampled by the k-mers. We found a strong linear relationship between Ba31 coverage and BCerG31 coverage within $B$. anthracis genome subsamples 
305 (Pearson's Correlation $r=0.99, p<0.001$, Supplementary Figure 2). As expected, the same 306 relationship did not appear when we subsampled non-B. anthracis BCerG members (Pearson's 307 Correlation $r=0.74, p<0.001$, Supplementary Figure 3 ). However, we did see a small number 308 of Ba31 k-mers detected, which we suspected were due to random errors introduced by lllumina 309 sequencing (Figure 4). The counts of false positive Ba31 k-mers scaled with the approximate 310 genetic distance to $B$. anthracis (as measured by mash (Ondov et al., 2016)) (Supplementary 311 Figure 4). We simulated synthetic data for a group of BCerG strains most closely related to $B$. 312 anthracis (Figure 3). We developed a linear regression model to relate BCerG k-mer coverage 313 and sequencing errors based on this group (Figure 4). For every unit of BCerG31 k-mer

A "specialist" model to interpret patterns of $B$. anthracis genetic signatures in metagenome samples

318 In real metagenome samples $B$. anthracis, if present, may only account for a low proportion of the total reads and may also be mixed with higher proportions of closely related BCerG strains. We sought to use the k-mer sets developed in the previous sections and knowledge of the lef gray zone coverage and BCerG false positive rate to interpret both synthetic and real metagenome datasets. The logic for assignment is shown in Table 1 and Supplementary Figure 5.

324

325

For our synthetic dataset we mixed low coverage B. anthracis with higher coverages of BCerG sequence data (see methods). We calculated the BCerG31 and Ba31 coverages for each mixture. Based on the BCerG sequence error model, we calculated the $99 \%$ count of Ba31 signatures predicted to be present by sequencing error under the assumption that there was no $B$. anthracis present and that all BCerG were drawn from the most closely related clade (Figure 3). We also reported whether the Ba31 coverage lay in or above the gray zone (Table 2, Supplementary File 2, Supplementary Figure 6). When B. anthracis was below $0.003 x$ genome coverage (approximately 16,000 bp), we could not distinguish its presence from errors produced in the absence of $B$. cereus. As expected, we found that the level of BCerG coverage determined the lower limit to differentiate genuine Ba31 hits from sequencing errors. At $75 x$ $B C$ erG coverage the required $B$. anthracis coverage to differentiate Ba31 matches from sequencing errors doubled to $0.006 \mathrm{x}$. The threshold for accurate detection was further raised to $0.01 \times$ B. anthracis genome coverage at $100 \times$ BCerG coverage.

In contrast, when the samples were classified using KrakenHLL(Breitwieser \& Salzberg, 2018), an accurate generalist program based on 31-mers, we found that all were predicted to contain $B$. anthracis, including negative controls (Table 2). The $B$. anthracis calls were made because of the sequence errors from the high coverage BCerG genomes.

Finally, we tested our model against the NYC dataset (Table 3, Supplementary File 2). All 1,458 samples were negative for lef31, in line with the conclusion reached from re-analysis of the dataset that $B$. anthracis was absent from the NY subway (Mason, 2015). We found that 1,367 of the 1,458 samples had at least one BCerG31 k-mer match and, of these, 1,085 contained at least one Ba31 match. We identified 34 samples with Ba31 counts above the 99\% 
349 threshold predicted by the BCerG coverage. These samples did not include the two (P00134 350 and P00497), previously flagged as B. anthracis positive (Afshinnekoo et al., 2015a) (Table 3). KrakenHLL also classified each these 34 samples as positive for $B$. anthracis.

\section{Discussion}

354 In this work we have described a significant update to a $B$. anthracis specific 31-mer set that was introduced in earlier blog posts (Petit et al., 2015; Minot et al., 2015) and we have shown how this set can be used to interpret $B$. anthracis specific signatures in Illumina metagenome samples. We chose to use k-mer-based signatures for the ease and speed of computation, with the length of $31 \mathrm{nt}$ selected as it was identified as the shortest likely to be unique across bacteria datasets (Koslicki \& Falush, 2016).

360

Some species present unusual challenges for metagenome identification. There is no consistently applied definition for the boundary that divides bacterial species based on DNA sequence identity and in some cases the presence or absence of mobile elements like plasmids and phages are required for speciation. $B$. anthracis is closely related to non-biothreat species and acquires its enhanced virulence from genes on mobile plasmids. Such species can be hard to model using "generalist" programs (such as Kraken) that attempt to classify every read in the dataset into one of thousands of taxonomic groups. We use a "specialist" approach aiming to solve a narrow problem that can be used to augment the predictions of generalist software. Specialist analyses can take advantage of unique features of the system and can also afford more effort in the curation of training data. In this case, we designed 31-mer signature sets based on comparison of hundreds of complete Bacillus genomes and we incorporated knowledge of false positive k-mers likely to be produced by close relatives of $B$. anthracis to develop a 'worst case' linear regression model to differentiate $B$. anthracis from sequencing errors. We also used the fact that the presence of a specific gene (lef) was diagnostic for anthrax. In designing our k-mer sets we encountered some rare cases of taxonomic misassignment in public datasets and were able to take corrective action (Figure 3). Generalist programs also rely on the same taxonomy and reference sequence databases, but it is harder to detect small errors that lead to mis-assignments when done on a large scale (Nasko et al., 2018). If we were to attempt approaches to specifically detect other known $B$. cereus strains that contain pXO1(Hoffmaster et al., 2004; Klee et al., 2010), we would have to develop and test new k-mer sets based on their unique chromosomal SNPs. Although we concentrate here on $B$. anthracis and BCerG, specialist methods could also be developed for other bacterial pathogens (e.g Yersinia pestis and Shigella sonnei) using a similar strategy of accounting for possible nonpathogen close relatives in the sample and the diagnostic presence of high consequence virulence genes acquired by horizontal transfer.

Even when a specialized algorithm has been developed, judgement is still required in interpreting results. In the case of the Bacillus genomes in particular, DNA extraction biases may affect results in ways we cannot assess without empirical experiments. We can't tell what proportion of the DNA came from lysis-resistant spores and what proportion was from the more fragile vegetative state, and how this balance might vary between strains across environments. 
392

393

394

395

396

397

398

399

400

401

402

403

404

405

406

407

408

409

410

411

412

413

414

415

416

417

418

419

420

421

422

423

424

425

426

427

428

429

430

431

432

433

434

Similarly, using a different sequencing technology, such the Pacific Biosystems SMRT system, with a different error profile, would require recalibration of the model.

Our reanalysis of the NYC data (Afshinnekoo et al., 2015a) showed that there was no direct evidence for the lethal factor k-mers in the metagenome samples (Supplementary File 2). This confirms other work (Mason, 2015; Minot et al., 2015; McIntyre et al., 2017), and together with the low prior probability of encountering $B$. anthracis in New York City, suggests that the samples taken were all negative for anthrax. The two samples originally flagged as possibly positive (Table 3) fell under case 4 (Table 1), as did 1,049 out of the other 1,456 samples. There were 373 samples with no Ba31 k-mer matches. These are all most likely true negatives, although, as we showed in the synthetic dataset, high BCerG coverage can mask the signal of low coverage $B$. anthracis (Table 2). To get a true negative would theoretically involve sequencing every cell in the sample (assuming perfectly efficient DNA preparation), which is impossible currently for all but the simplest communities. The limit of detection will be a complex calculation that involves the amount of DNA sequence generated and the complexity of the microbial community. Negative (and positive) calls ultimately have to be supported through sensitive detection assays such as PCR and/or culture.

We identified 34 samples above the BCerG thresholds for our model (Table 3). All the samples fell under case 3 except a single sample which fell under case 2 (Table 1). An outlier of case 3 samples, P00981, taken from a metal handrail on the A train route(Afshinnekoo et al., 2015a), had high Ba31 counts $(n=20,079)$. As we collect more genomes of $B$. cereus group we may see more Ba31 k-mers in BCerG genomes. These samples may contain members of yet unencountered lineages more closely related to $B$. anthracis than previously seen, or possibly the result of recent recombination between $B$. anthracis and $B$. cereus genomes (although the latter has not been reported). It is important that these strains are isolated, sequenced and added to public databases to iteratively improve pathogen detection. The single case 2 sample, P00738 (Table 3), was also on a metal handrail from the A train route, although sampled 3 days earlier than P00981. This sample was possibly the most problematic because the Ba31 counts were in the gray zone, meaning there was not enough coverage to rule out lef being present. Most likely, this sample contained another near- $B$. anthracis strain, but case 2 samples should be a priority for retesting by culture and PCR methods.

\section{Conclusions}

If $B$. anthracis, or another BCerG strain containing $\mathrm{pXO1}$, is present in a shotgun metagenome sample at high genome coverage, identification of lef k-mers is a strong signal for the likely presence of anthrax-causing bacteria. We showed that using a $B$. anthracis specific k-mer set alone to call the presence of $B$. anthracis produced many false positive calls because sequencing errors of common co-resident BCerG bacteria. We developed models to partition cases that contained evidence of possible low coverage $B$. anthracis, accounting for $B$. cereus coverage. However, in simulations, we showed that false negative results can arise when the BCerG coverage is high. Reanalysis of the NYC subway metagenome study confirmed the absence of $B$. anthracis containing lef but we found evidence in at least two samples of BCerG strains that contained what were considered $B$. anthracis specific sequences. Culturing strains 
435 such as these, genome sequencing and sharing to the public domain will help improve $B$.

436 anthracis detection in metagenome shotgun samples.

\section{Acknowledgements}

438 Thanks to Sam Minot, Chris Greenfield, Chris Mason and his group for discussion following our 439 original blog post.

440

\section{References}

442

443 Ackelsberg J., Rakeman J., Hughes S., Petersen J., Mead P., Schriefer M., Kingry L.,

444 Hoffmaster A., Gee JE. 2015. Lack of Evidence for Plague or Anthrax on the New York City

445 Subway. Cell systems 1:4-5.

Afshinnekoo E., Meydan C., Chowdhury S., Jaroudi D., Boyer C., Bernstein N., Maritz JM.,

Reeves D., Gandara J., Chhangawala S., Ahsanuddin S., Simmons A., Nessel T.,

Sundaresh B., Pereira E., Jorgensen E., Kolokotronis S-O., Kirchberger N., Garcia I., Magalhaes TR., Boone B., Jones AL., Muth TR., Paolantonio KS., Alter E., Schadt EE., Garbarino J., Prill RJ., Carlton JM., Levy S., Mason CE. 2015a. Geospatial Resolution of Human and Bacterial Diversity with City-Scale Metagenomics. Cell Systems. DOI: 10.1016/j.cels.2015.01.001.

Afshinnekoo E., Meydan C., Chowdhury S., Jaroudi D., Boyer C., Bernstein N., Maritz JM., P., Walsh M., O’Mullan GD., Tighe S., Dudley JT., Dunaif A., Ennis S., O’Halloran E., Magalhaes TR., Boone B., Jones AL., Muth TR., Paolantonio KS., Alter E., Schadt EE., Garbarino J., Prill RJ., Carlton JM., Levy S., Mason CE. 2015b. Modern Methods for Delineating Metagenomic Complexity. Cell systems 1:6-7. 
463 Bragg TS., Robertson DL. 1989. Nucleotide sequence and analysis of the lethal factor gene (lef) 464 from Bacillus anthracis. Gene 81:45-54.

465 Breitwieser FP., Salzberg SL. 2018. KrakenHLL: Confident and fast metagenomics classification $466 \quad$ using unique k-mer counts. bioRxiv:262956. DOI: 10.1101/262956.

467 Brown CT., Olm MR., Thomas BC., Banfield JF. 2016. Measurement of bacterial replication 468 rates in microbial communities. Nature biotechnology 34:1256-1263.

469 Cachat E., Barker M., Read TD., Priest FG. 2008. A Bacillus thuringiensis strain producing a 470 polyglutamate capsule resembling that of Bacillus anthracis. FEMS microbiology letters $471 \quad 285: 220-226$.

472 Camacho C., Coulouris G., Avagyan V., Ma N., Papadopoulos J., Bealer K., Madden TL. 2009. $473 \quad$ BLAST+: architecture and applications. BMC bioinformatics 10:421.

474 Carlson CJ., Getz WM., Kausrud KL., Cizauskas CA., Blackburn JK., Bustos Carrillo FA., 475 Colwell R., Easterday WR., Ganz HH., Kamath PL., Økstad OA., Turner WC., Kolstø A-B., 476 Stenseth NC. 2018. Spores and soil from six sides: interdisciplinarity and the environmental 477 biology of anthrax ( Bacillus anthracis ) : The environmental biology of Bacillus anthracis. $478 \quad$ Biological Reviews 424:329.

479 Dixon TC., Meselson M., Guillemin J., Hanna PC. 1999. Anthrax. The New England journal of $480 \quad$ medicine $341: 815-826$.

481 Gordon A., Hannon GJ. 2010. Fastx-toolkit. Computer program distributed by the author, 482 website http://hannonlab. cshl. edu/fastx_toolkit/index. html [accessed 2014--2015]. 483 Helgason E., Okstad OA., Caugant DA., Johansen HA., Fouet A., Mock M., Hegna I., Kolstø 484 AB. 2000. Bacillus anthracis, Bacillus cereus, and Bacillus thuringiensis--one species on 485 the basis of genetic evidence. Applied and environmental microbiology 66:2627-2630. 486 Hoffmann C., Zimmermann F., Biek R., Kuehl H., Nowak K., Mundry R., Agbor A., Angedakin 487 S., Arandjelovic M., Blankenburg A., Brazolla G., Corogenes K., Couacy-Hymann E., 488 Deschner T., Dieguez P., Dierks K., Düx A., Dupke S., Eshuis H., Formenty P., Yuh YG., 
Goedmakers A., Gogarten JF., Granjon A-C., McGraw S., Grunow R., Hart J., Jones S.,

Huang W., Li L., Myers JR., Marth GT. 2012. ART: a next-generation sequencing read Junker J., Kiang J., Langergraber K., Lapuente J., Lee K., Leendertz SA., Léguillon F., Leinert V., Löhrich T., Marrocoli S., Mätz-Rensing K., Meier A., Merkel K., Metzger S., Murai M., Niedorf S., De Nys H., Sachse A., van Schijndel J., Thiesen U., Ton E., Wu D., Wieler LH., Boesch C., Klee SR., Wittig RM., Calvignac-Spencer S., Leendertz FH. 2017. Persistent anthrax as a major driver of wildlife mortality in a tropical rainforest. Nature 548:82-86.

Hoffmaster AR., Ravel J., Rasko DA., Chapman GD., Chute MD., Marston CK., De BK., Sacchi CT., Fitzgerald C., Mayer LW., Maiden MCJ., Priest FG., Barker M., Jiang L., Cer RZ., Rilstone J., Peterson SN., Weyant RS., Galloway DR., Read TD., Popovic T., FraserLiggett CM. 2004. Identification of anthrax toxin genes in a Bacillus cereus associated with an illness resembling inhalation anthrax. Proceedings of the National Academy of Sciences of the United States of America 101:8449-8454. simulator. Bioinformatics 28:593-594.

Keim PS., Wagner DM. 2009. Humans and evolutionary and ecological forces shaped the phylogeography of recently emerged diseases. Nature reviews. Microbiology 7:813-821.

Klee SR., Brzuszkiewicz EB., Nattermann H., Brüggemann H., Dupke S., Wollherr A., Franz T., Pauli G., Appel B., Liebl W., Couacy-Hymann E., Boesch C., Meyer F-D., Leendertz FH., Ellerbrok H., Gottschalk G., Grunow R., Liesegang H. 2010. The genome of a Bacillus isolate causing anthrax in chimpanzees combines chromosomal properties of $B$. cereus with B. anthracis virulence plasmids. PloS one 5:e10986.

Konstantinidis KT., Tiedje JM. 2005. Genomic insights that advance the species definition for prokaryotes. Proceedings of the National Academy of Sciences of the United States of America 102:2567-2572.

Koslicki D., Falush D. 2016. MetaPalette: a k-mer Painting Approach for Metagenomic 
515 Taxonomic Profiling and Quantification of Novel Strain Variation. mSystems 1. DOI:

$516 \quad 10.1128 / m S y s t e m s .00020-16$.

517 Li H., Durbin R. 2009. Fast and accurate short read alignment with Burrows-Wheeler transform.

518 Bioinformatics 25:1754-1760.

519 Li H., Handsaker B., Wysoker A., Fennell T., Ruan J., Homer N., Marth G., Abecasis G., Durbin 520 R., 1000 Genome Project Data Processing Subgroup. 2009. The Sequence Alignment/Map 521 format and SAMtools. Bioinformatics 25:2078-2079.

522 Marçais G., Kingsford C. 2011. A fast, lock-free approach for efficient parallel counting of 523 occurrences of k-mers. Bioinformatics 27:764-770.

524 Mason C. 2015.The long road from Data to Wisdom, and from DNA to Pathogen. Available at 525 https://www.microbe.net/2015/02/17/the-long-road-from-data-to-wisdom-and-from-dna-to526 pathogen/ (accessed December 18, 2017).

527 Mclntyre ABR., Ounit R., Afshinnekoo E., Prill RJ., Hénaff E., Alexander N., Minot SS., Danko 528 D., Foox J., Ahsanuddin S., Tighe S., Hasan NA., Subramanian P., Moffat K., Levy S., 529 Lonardi S., Greenfield N., Colwell RR., Rosen GL., Mason CE. 2017. Comprehensive 530 benchmarking and ensemble approaches for metagenomic classifiers. Genome biology $531 \quad 18: 182$

532 Minot SS., Greenfield N., Afshinnekoo E., Mason CE. 2015.Anthrax Marker Panel. Available at 533 https://science.onecodex.com/bacillus-anthracis-panel/ (accessed December 19, 2017).

534 Nasko DJ., Koren S., Phillippy AM., Treangen TJ. 2018. RefSeq database growth influences the 535 accuracy of k-mer-based species identification. bioRxiv:304972. DOI: 10.1101/304972.

536 Ondov BD., Treangen TJ., Melsted P., Mallonee AB., Bergman NH., Koren S., Phillippy AM. 537 2016. Mash: fast genome and metagenome distance estimation using MinHash. Genome 538 biology 17:132.

539 Pannucci J., Okinaka RT., Williams E., Sabin R., Ticknor LO., Kuske CR. 2002. DNA sequence 540 conservation between the Bacillus anthracis pXO2 plasmid and genomic sequence from 
$541 \quad$ closely related bacteria. BMC genomics 3:34

542 Petit RA III., Ezewudo M., Joseph SJ., Read TD. 2015.Searching for anthrax in the New York

543 City subway metagenome. Available at $h t t p: / / d x . d o i . o r g / 10.5281 /$ zenodo. 17158 (accessed

$544 \quad$ December 18, 2017). DOI: 10.5281/zenodo.17158.

545 Quast C., Pruesse E., Yilmaz P., Gerken J., Schweer T., Yarza P., Peplies J., Glöckner FO.

546 2013. The SILVA ribosomal RNA gene database project: improved data processing and $547 \quad$ web-based tools. Nucleic acids research 41:D590-6.

548 Quinlan AR., Hall IM. 2010. BEDTools: a flexible suite of utilities for comparing genomic 549 features. Bioinformatics 26:841-842.

550 Rasko DA., Rosovitz MJ., Økstad OA., Fouts DE., Jiang L., Cer RZ., Kolstø A-B., Gill SR., 551 Ravel J. 2007. Complete sequence analysis of novel plasmids from emetic and periodontal 552 Bacillus cereus isolates reveals a common evolutionary history among the $B$. cereus-group 553 plasmids, including Bacillus anthracis pX01. Journal of bacteriology 189:52-64.

554

Read TD., Salzberg SL., Pop M., Shumway M., Umayam L., Jiang L., Holtzapple E., Busch JD., 555 Smith KL., Schupp JM., Solomon D., Keim P., Fraser CM. 2002. Comparative genome 556 sequencing for discovery of novel polymorphisms in Bacillus anthracis. Science 296:2028-

557 2033.

558

Segata N., Waldron L., Ballarini A., Narasimhan V., Jousson O., Huttenhower C. 2012.

559 Metagenomic microbial community profiling using unique clade-specific marker genes.

$560 \quad$ Nature methods 9:811-814.

561 Zwick ME., Joseph SJ., Didelot X., Chen PE., Bishop-Lilly KA., Stewart AC., Willner K., Nolan

562 N., Lentz S., Thomason MK., Sozhamannan S., Mateczun AJ., Du L., Read TD. 2012.

563 Genomic characterization of the Bacillus cereus sensu lato species: backdrop to the 564 evolution of Bacillus anthracis. Genome research 22:1512-1524. 


\section{Table $\mathbf{1}$ (on next page)}

Potential outcomes of $\mathrm{B}$. anthracis detection, given matches to the Ba31 set in a shotgun metagenome dataset

This table discusses the interpretation of four cases when Ba31 k-mer matches are found in the dataset. Columns 1-3 are; lef31 match; whether Ba31 coverage is in the Gray Zone; and whether Ba31 coverage is above the $99 \%$ of the error model based on BCerG coverage. 


\begin{tabular}{|c|c|c|c|c|}
\hline Case & Lef31 & Gray Zone & $\begin{array}{c}\text { Exceeds } \\
99 \% \text { P.I. }\end{array}$ & Interpretation \\
\hline 1 & yes & yes or no & yes or no & $\begin{array}{r}\text { Evidence of lethal factor gene, could be } B . \\
\text { anthracis or a B. cereus strain carrying the } \\
\text { pXO1 plasmid. }\end{array}$ \\
\hline 2 & no & yes & yes & $\begin{array}{r}\text { Possible B. anthracis or closely related strain } \\
\text { based on high Ba31 counts but genome } \\
\text { coverage too low to guarantee seeing the lef } \\
\text { gene. Requires more sequence coverage } \\
\text { and/or validation by PCR or other methods. }\end{array}$ \\
\hline 3 & no & no & yes & $\begin{array}{c}\text { Ba31 matches exceed what is expected by the } \\
\text { BCerG error model, but are at a level of genome } \\
\text { coverage at which lethal factor should have } \\
\text { been detected. Most likely explanation is } B . \\
\text { anthracis strain cured of pXO1 or unsequenced } \\
\text { lineage closely related to B. anthracis. }\end{array}$ \\
\hline 4 & no & yes or no & no & $\begin{array}{l}\text { Most likely scenario is that BCerG background } \\
\text { produced Ba31 k-mers through random errors } \\
\text { but impossible to also rule out presence of low } \\
\text { coverage B. anthracis }\end{array}$ \\
\hline
\end{tabular}

1

${ }^{1}$ Prediction Interval

3 


\section{Table 2 (on next page)}

\section{Artificial mixtures of low coverage $B$. anthracis and high coverage $B$. cereus}

This table shows some key results from more than 300 artificial mixtures of $B$. anthracis and $B$. cereus sequences created to test our specialized model (Supplementary File 1). The table includes three $B$. anthracis coverages for each $B$. cereus coverage. The $B$. anthracis simulated coverages represent the minimum $B$. anthracis coverage, the coverage at which $B$. anthracis was detectable, and the maximum $B$. anthracis coverage. The first two columns are the coverage in the artificial mixtures of $B$. cereus and $B$. anthracis genomes, respectively. The third column is the observed BCerG31 k-mer coverage. Columns 4-6 are the observed number of Ba31 k-mers, the expected number of Ba31 k-mers based on the BCerG31 coverage (see Figure 4 ) and the $99 \%$ prediction interval of the model, which we take as an indicative worst case threshold. The seventh column summarizes whether the observed Ba31 is greater than the $99 \%$ P.I. The eighth column is whether the Ba31 coverage is in the "gray zone" $(<0.18 x$ coverage). "No" means the Ba31 exceeds the threshold (note it is possible for the Ba31 coverage to be at gray zone level but still have a positive match to a lef31k-mer). The final column shows whether KrakenHLL (Breitwieser \& Salzberg, 2018) run on the sample predicted the presence of $B$. anthracis.

This table shows that false positives k-mers resulting from high BCerG coverage limit the detection of $B$. anthracis k-mers (Ba31) in mixed cultures. Below 0.006x (75x-fold $B$. cereus) and $0.01 x$ ( $100 \mathrm{x}$-fold $B$. cereus) $B$. anthracis genome coverages, true positive Ba31 matches cannot be differentiated from false positive matches. KrakenHLL predicted $B$. anthracis to be present even when it was not because of the background BCerG genomes coverage. 


\begin{tabular}{|c|c|c|c|c|c|c|c|c|}
\hline \multicolumn{2}{|c|}{$\begin{array}{c}\text { Artificial Genome } \\
\text { Coverage }\end{array}$} & \multirow[b]{2}{*}{$\begin{array}{l}\text { BCerG31 } \\
\text { Coverage }\end{array}$} & \multicolumn{3}{|c|}{ Ba31 Count } & \multirow[b]{2}{*}{$\begin{array}{l}\text { Exceeds } \\
99 \% \text { P.I. }{ }^{1}\end{array}$} & \multirow[b]{2}{*}{$\begin{array}{l}\text { lef31 } \\
\text { Gray } \\
\text { Zone }\end{array}$} & \multirow[b]{2}{*}{$\begin{array}{c}\text { Kraken } \\
\text { HLL }\end{array}$} \\
\hline B. cereus & B. anthracis & & Observed & $\begin{array}{c}\text { Model } \\
\text { Fit }\end{array}$ & $\begin{array}{c}\text { Model } \\
\text { Upper } \\
99 \% \text { P.I. }{ }^{1}\end{array}$ & & & \\
\hline $0 x$ & $0.001 x$ & 0.00002 & 10 & 1 & 331 & No & Yes & Yes \\
\hline $0 x$ & $0.003 x$ & 0.00245 & 346 & 1 & 332 & Yes & Yes & Yes \\
\hline $0 x$ & $0.2 x$ & 0.123 & 25,396 & 21 & 352 & Yes & No & Yes \\
\hline $1 x$ & $0 x$ & 0.593 & 99 & 102 & 433 & No & Yes & Yes \\
\hline $1 x$ & $0.003 x$ & 0.610 & 444 & 104 & 437 & Yes & Yes & Yes \\
\hline $1 x$ & $0.2 x$ & 0.727 & 25,627 & 125 & 456 & Yes & No & Yes \\
\hline $5 x$ & $0 x$ & 3.048 & 487 & 524 & 855 & No & Yes & Yes \\
\hline $5 x$ & $0.003 x$ & 3.060 & 919 & 526 & 857 & Yes & Yes & Yes \\
\hline $5 x$ & $0.2 x$ & 3.155 & 25,502 & 542 & 874 & Yes & No & Yes \\
\hline $10 x$ & $0 x$ & 6.115 & 1,050 & 1,051 & 1,382 & No & Yes & Yes \\
\hline $10 x$ & $0.004 x$ & 6.100 & 1,531 & 1,048 & 1,379 & Yes & Yes & Yes \\
\hline $10 x$ & $0.2 x$ & 6.450 & 26,346 & 1,074 & 1,405 & Yes & No & Yes \\
\hline $25 x$ & $0 x$ & 15.277 & 2,516 & 2,625 & 2,957 & No & Yes & Yes \\
\hline $25 x$ & $0.004 x$ & 15.174 & 3,075 & 2,608 & 2,939 & Yes & Yes & Yes \\
\hline $25 x$ & $0.2 x$ & 15.339 & 27,536 & 2,636 & 2,967 & Yes & No & Yes \\
\hline $50 x$ & $0 x$ & 30.381 & 5,058 & 5,221 & 5,552 & No & Yes & Yes \\
\hline $50 x$ & $0.005 x$ & 30.438 & 5,726 & 5,231 & 5,562 & Yes & Yes & Yes \\
\hline $50 x$ & $0.2 x$ & 30.595 & 29,766 & 5,257 & 5,589 & Yes & No & Yes \\
\hline $75 x$ & $0 x$ & 45.753 & 7,323 & 4,530 & 8,194 & No & Yes & Yes \\
\hline $75 x$ & $0.006 x$ & 45.699 & 8,351 & 7,853 & 8,184 & Yes & Yes & Yes \\
\hline
\end{tabular}




\begin{tabular}{|l|l|c|c|c|c|c|c|c|}
\hline $75 x$ & $0.2 x$ & 45.859 & 31,971 & 7,881 & 8,212 & Yes & No & Yes \\
\hline $100 x$ & $0 x$ & 60.926 & 9,633 & 10,470 & 10,801 & No & Yes & Yes \\
\hline $100 x$ & $0.01 x$ & 60.958 & 11,020 & 10,475 & 10,807 & Yes & Yes & Yes \\
\hline $100 x$ & $0.2 x$ & 61.093 & 33,761 & 10,498 & 10,830 & Yes & No & Yes \\
\hline
\end{tabular}

2

31 Prediction Interval

4

5 


\section{Table 3 (on next page)}

Reanalysis of NYC subway metagenome sequencing

We counted Ba31, BCerG31 and lef31 k-mers in 1,458 NYC subway metagenomic samples (Afshinnekoo et al., 2015). The table is a breakdown of samples that were within the gray zone and/or had Ba31 matches that exceed the 99\% prediction interval. Columns 2-8 display the same data types as columns 3-9 in Table 2. The additional lef column shows whether lef31 matches were identified or not. The final column provides the outcome case of the sample (Table 1). This table presents 4 samples excerpted from the complete results for all samples (Supplementary File 2). There is one sample within the gray zone (P00738), two from the original study (P00134 and P00497) and an outlier of samples which exceed the 99\% prediction interval (P00981). 


\begin{tabular}{|c|c|c|c|c|c|c|c|c|c|}
\hline \multirow[b]{2}{*}{ Sample } & \multirow[b]{2}{*}{$\begin{array}{l}\text { BCerG31 } \\
\text { Coverage }\end{array}$} & \multicolumn{3}{|c|}{ Ba31 Count } & \multirow[b]{2}{*}{$\begin{array}{l}\text { Exceeds } \\
99 \% \text { P.I. }\end{array}$} & \multirow[b]{2}{*}{$\begin{array}{l}\text { Gray } \\
\text { Zone }\end{array}$} & \multirow[b]{2}{*}{$\begin{array}{c}\text { Kraken } \\
\text { HLL }\end{array}$} & \multirow[b]{2}{*}{ lef } & \multirow[b]{2}{*}{$\begin{array}{c}\text { Outcome } \\
\text { Case }\end{array}$} \\
\hline & & $\begin{array}{c}\text { Obser } \\
\text { ved }\end{array}$ & $\begin{array}{l}\text { Model } \\
\text { Fit }\end{array}$ & $\begin{array}{c}\text { Model } \\
\text { Upper } \\
99 \% \text { P.I. }{ }^{2}\end{array}$ & & & & & \\
\hline P001341 & 19.71 & 2,755 & 3,387 & 3,718 & No & No & Yes & No & 4 \\
\hline P004971 & 4.05 & 953 & 696 & 1,027 & No & No & Yes & No & 4 \\
\hline P00981 & 1.32 & 20,079 & 226 & 558 & Yes & No & Yes & No & 3 \\
\hline P00738 & 0.002 & 396 & 1 & 331 & Yes & Yes & Yes & No & 2 \\
\hline \multicolumn{10}{|l|}{2} \\
\hline $1 \mathrm{Sa}$ & ples previo & I & ied as c & ntaining $B$. & inthracis & & & & \\
\hline $2 \operatorname{Pr}$ & iction Inter & & & & & & & & \\
\hline
\end{tabular}


Figure 1 (on next page)

Flowchart of strategy for primer design

We developed a strategy for selecting the Ba31 and BCerG31 (A) and lef31 (B) k-mer sets. In A) the outgroup is determined by the k-mer set. For Ba31, the outgroup was comprised of all the non-B. anthracis genomes; for BCerG31, it consisted of all non-B. cereus group genomes. 
A

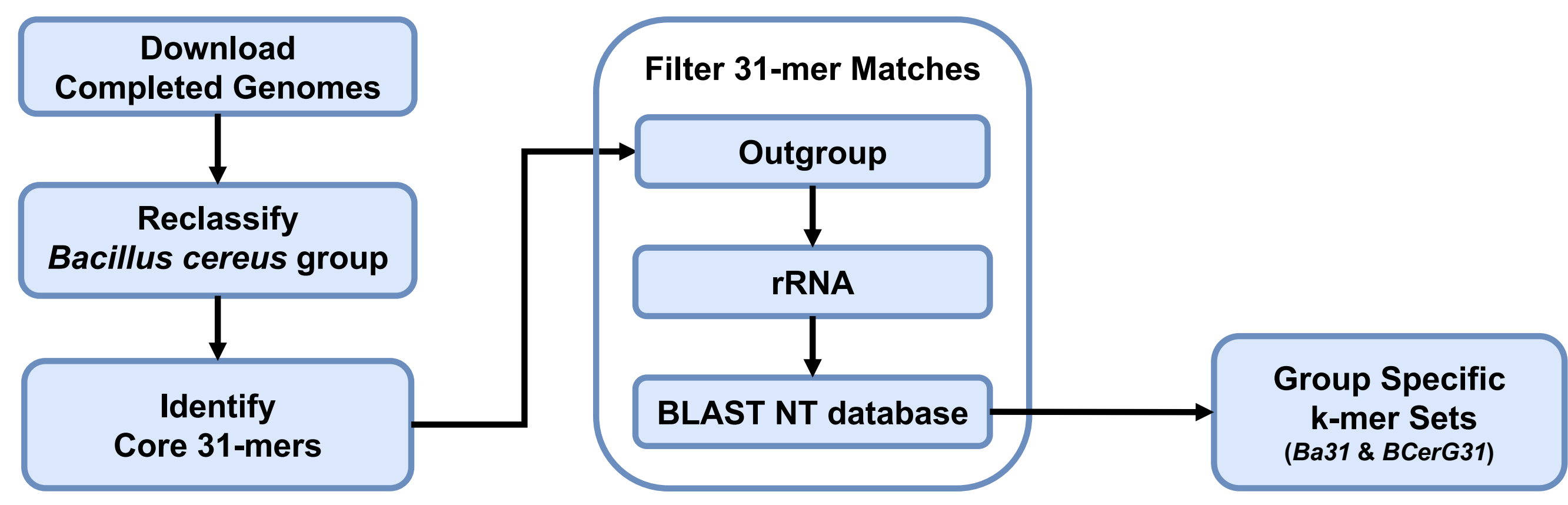

B

Download pX01 Plasmids

\section{Extract}

Lethal Factor Gene
Identify Unique Set of 31-mers
Lethal Factor Gene k-mer Set (lef31) 


\section{Figure 2 (on next page)}

\section{Limit of detection for lethal factor gene k-mers (lef31)}

164 B. anthracis sequencing projects were subsampled to different levels of genome coverage, with 100 random subsamples obtained for each coverage level. Our ability to detect the lethal factor gene is assessed by considering the number of these subsamples for which we find at least one lef31 k-mer hit. Two thresholds - $95 \%$ and $100 \%$ - were employed and are shown as colored series below.

The figure thus shows the percentage of the $B$. anthracis sequencing projects for which $95 \%$ (or $100 \%$ ) of the random subsamples contain at least one lef31 k-mer. Panel (A) shows results with respect to Ba31 kmer coverage while panel $(B)$ shows the corresponding results for BCerG coverage. The vertical dashed lines show the coverage limits for detection at the respective threshold levels. 
PeerJ

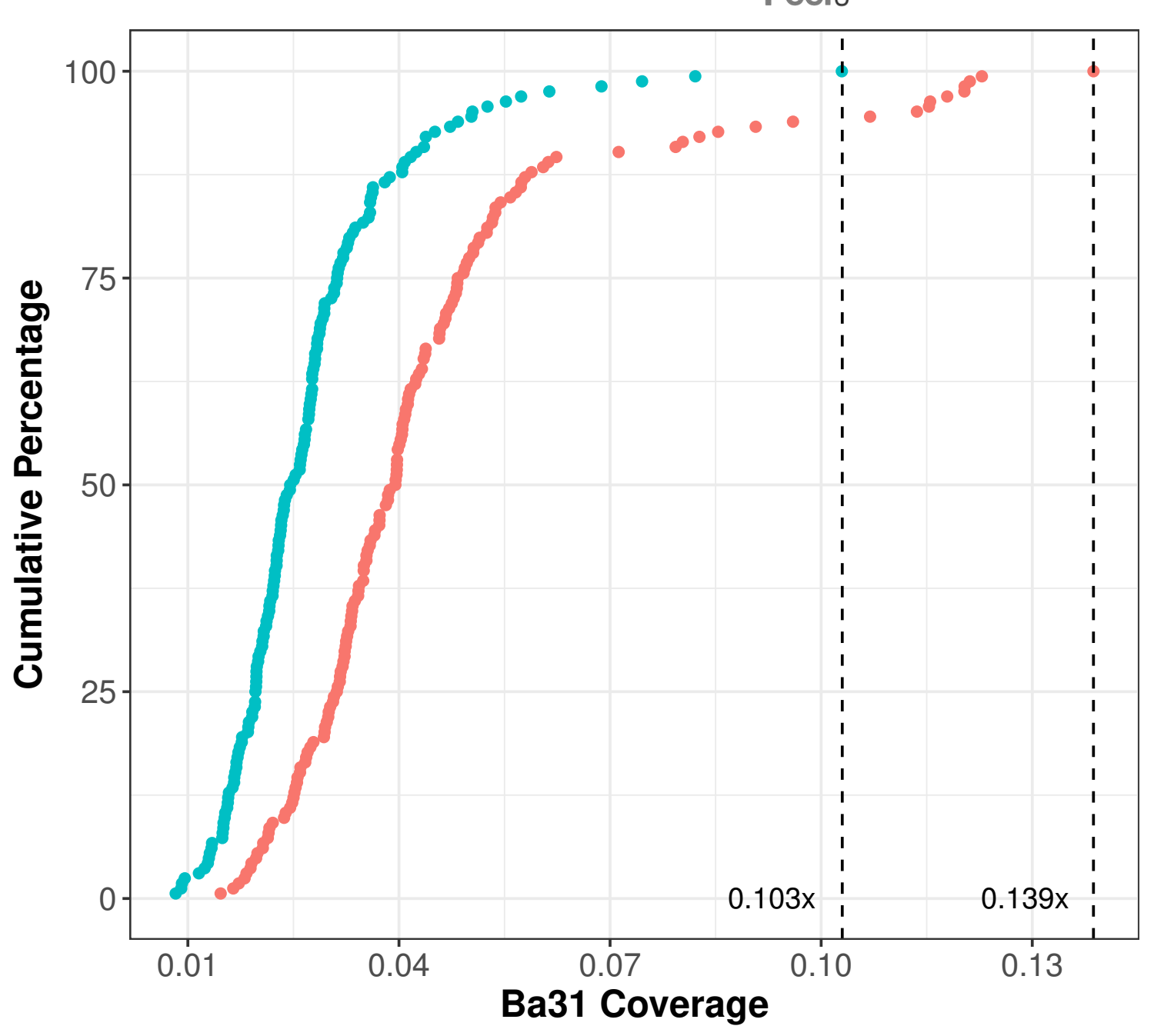

B Manuscript to be reviewed

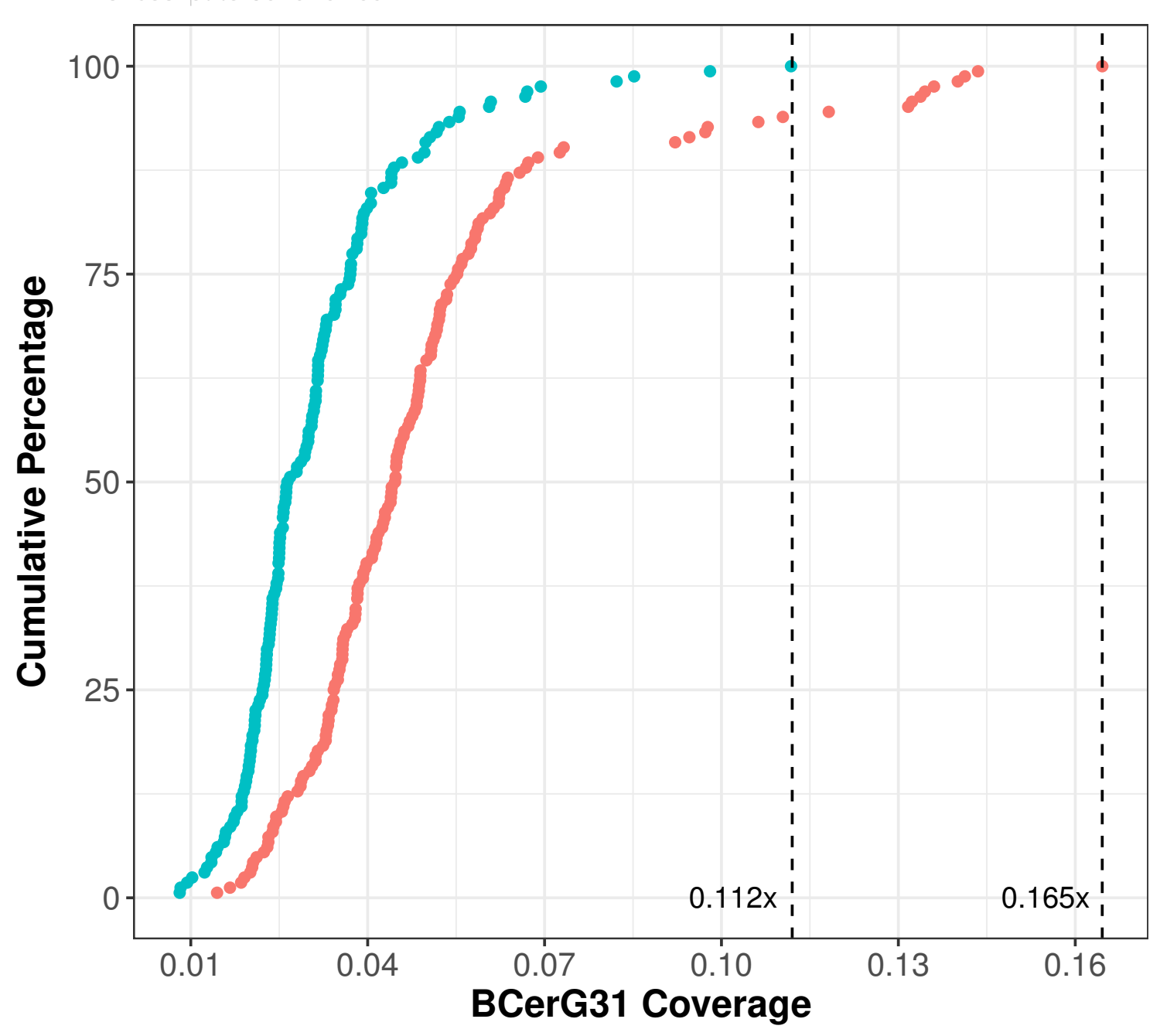




\section{Figure 3}

Unrooted phylogeny of BCerG genome assemblies used in the study after reclassifying BCerG strains

An unrooted phylogenetic representation of 140 BCerG genomes using Mashtree (v0.32, https://github.com//skatz/mashtree ). Genomes reclassified as BCerG members with mash (v2.0, (Ondov et al., 2016) ) are indicated with stars. The clade colored blue are B. cereus genomes closely related to $B$. anthracis that were used to model false positive results (Figure 4). 


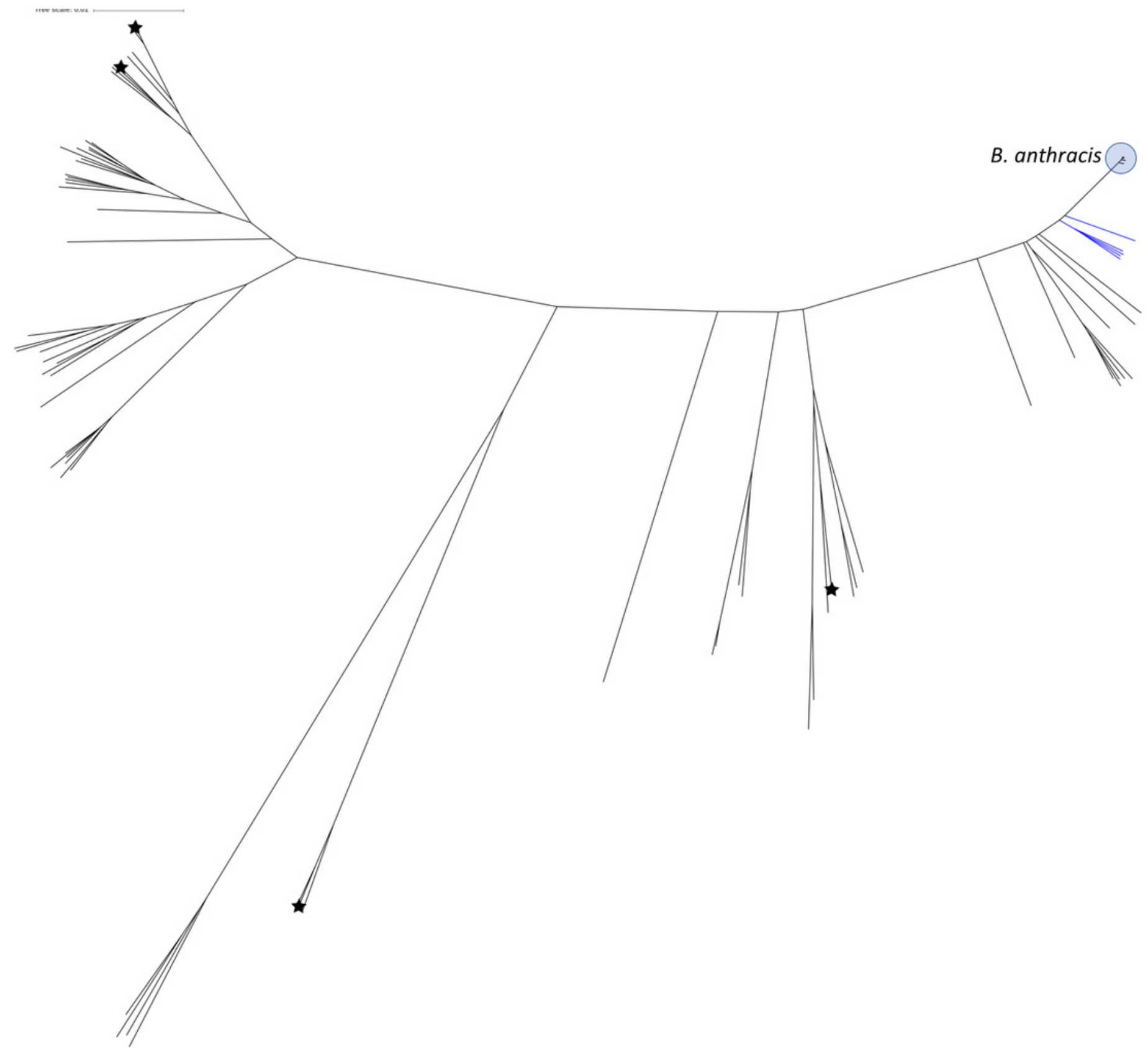




\section{Figure 4}

Linear regression model fit of BCerG coverage and false positive Ba31 counts

We created random synthetic FASTQ files based on BCerG chromosomes from the clade closest to $B$. anthracis (blue in Figure 3 ) at different genome coverages and counted the false positive Ba31 k-mers. Shown is the fit of a linear regression model with an intercept of 0, with BCerG31 coverage as the independent variable and the Ba31 false positive count as the dependent variable. The solid line shows the predicted values from the model, and the dashed line reflects the upper 99\% prediction interval for the parameters, which we use in the analyses above.

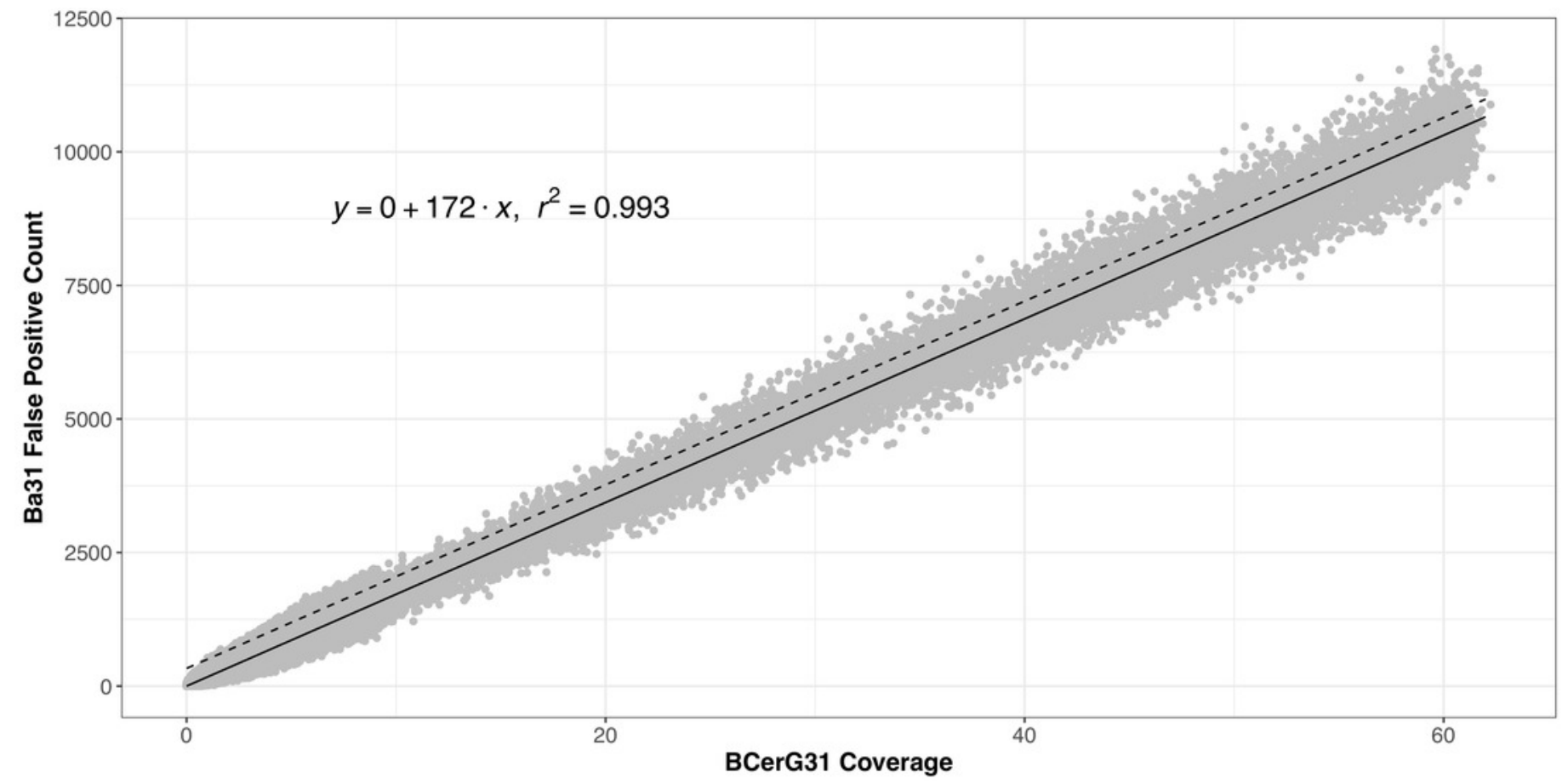

